\title{
Comportamiento del acero AISI-SAE 8615 frente al proceso de nitrocarburación ferrítica, utilizando urea-metano $1^{(\bullet)}$
}

\author{
M.I. Barrena ${ }^{(*)}$ y A. Castro ${ }^{(* *)}$
}

\begin{abstract}
Resumen El presente trabajo muestra el comportamiento del acero aleado de bajo contenido de carbono, AISISAE 8615 , cuando se somete a un proceso de nitrocarburación ferrítica, a la temperatura de $570{ }^{\circ} \mathrm{C}$ y un tiempo de $3 \mathrm{~h}$. La atmósfera reactiva ha sido generada mediante goteo variable de metanol y urea dentro del horno, utilizando distintas velocidades de flujo. Las capas nitrocarburadas fueron evaluadas mediante técnicas de microscopía óptica. Con el fin de conocer la extensión de las capas nitrocarburadas obtenidas, se realizaron medidas de microdureza y se determinaron los porcentajes de carbono en el proceso mediante espectroscopía de emisión. Se han encontrado las condiciones de flujo óptimo de la mezcla anteriormente comentada y se ha observado cómo su variación influye en el espesor de la capa nitrocarburada.
\end{abstract}

Palabras clave: Acero. Tratamientos termoquímicos. Nitrocarburación. Urea-Metanol.

\section{Behaviour of AISI-SAE 8615 steel in ferritic nitrocarburizing using urea-metanol}

\begin{abstract}
The present work shows the behaviour of low carbon alloyed steels as AISI-SAE 8615, after a ferritic nitrocarburizing process. Nitrocarburizing takes place at $570{ }^{\circ} \mathrm{C}$ for $3 \mathrm{~h}$ in an atmosphere by combustion of methanol and urea under different flow rates. Metallographic studies were performed by optical microscopy. Hardness profiles were measured and carbon percentages were also analized by emision spectrometry in order to determine the extension of the nitrocarburizing process. Optimal flow conditions have been found. The influence of the flow rate on the nitrocarburizing layer thickness has been also studied.
\end{abstract}

Keywords: Steel. Thermochemical treatments. Nitrocarburizing. Urea-Methanol.

\section{INTRODUCCIÓN}

Los procesos de nitrocarburación son tratamientos termoquímicos, que consisten en adicionar carbono y nitrógeno por difusión a través de la superficie del material a nitrocarburar (aleaciones férreas). Estos procesos se llevan a cabo a temperaturas elevadas dentro del campo ferrítico. Con el proceso de nitrocarburación, se pretende obtener en la superfi-

(•) Trabajo recibido el día 16 de junio de 1997.

(*) Dpto. de Ciencia de los Materiales e Ingeniería Metalúrgica. Facultad de Ciencias Químicas. Universidad Complutense de Madrid. Ciudad Universitaria. 28040-Madrid (España).

(**) Dpto. de Ingeniería Mecánica. Facultad de Ingeniería. Universidad Nacional de Colombia. Santa Fe de Bogotá (Colombia). cie del acero tratado, una fina capa blanca del orden de $\mu \mathrm{m}$, formada, principalmente, por nitrocarburos de hierro (fase $\varepsilon$ ), por debajo de la cual existe una zona de difusión que contiene nitrógeno disuelto y nitruros ferrosos.

Los procesos de nitrocarburación se realizan en medio líquido y en atmósfera. La nitrocarburación líquida se realiza en baños de sales fundidas que suelen contener cianuro como agente aportador de carbono y nitrógeno. Debido a los problemas de contaminación que presentan estos baños, se están sustituyendo por sales libres de cianuros. La nitrocarburación en atmósfera, se suele realizar utilizando mezclas de amoniaco, hidrocarburos y gases portadores (usualmente atmósferas endotérmicas o exotérmicas) en distintas proporciones. Las propiedades de las capas nitrocarburadas son similares independientemente del proceso. 
Las variables más importantes que afectan al proceso de nitrocarburación gaseosa son las condiciones de las atmósferas empleadas y de las reacciones que lo controlan (1). Las reacciones de este proceso se basan en la craquización de la urea [1] y del metanol [2].

$$
\begin{aligned}
& \mathrm{OC}\left(\mathrm{NH}_{2}\right)_{2} \Leftrightarrow \mathrm{N}_{2}+\mathrm{CO}+2 \mathrm{H}_{2} \\
& \mathrm{HOCH}_{3} \Leftrightarrow \mathrm{CO}+2 \mathrm{H}_{2}
\end{aligned}
$$

Desde el punto de vista de la nitrocarburación existe una analogía en el comportamiento de la urea y el amoniaco, pues ambos pueden incorporar nitrógeno al acero. Además, no existe precipitación de carbono durante la craquización que pudiera afectar al potencial de carbono de la atmósfera generada.

En la nitrocarburación gaseosa, la temperatura del horno ha de ser lo suficientemente baja como para mantener una estructura mayoritariamente ferrítica en el acero. Esta temperatura debe ser próxima a los $570{ }^{\circ} \mathrm{C}$, justo por debajo de la temperatura eutectoide del sistema Fe-C-N (Fig. 1). El tiempo de tratamiento puede oscilar entre 1 y $5 \mathrm{~h}$ (2).

Somers et al. (3) han estudiado la constitución de la capa formada (capa compuesta), mediante un modelo experimental realizado a $570{ }^{\circ} \mathrm{C}$, con una mezcla gaseosa constituida por $3 \% \mathrm{CO}, 53 \% \mathrm{NH}_{3}$ y $44 \% \mathrm{H}_{2}$. Inicialmente, se forma la fase $\gamma^{\prime}-$ $\mathrm{Fe}_{4}(\mathrm{~N}, \mathrm{C})_{1-\mathrm{x}}$ y sobre ésta comienza a formarse la fase $\varepsilon-\mathrm{Fe}_{2}(\mathrm{~N}, \mathrm{C})_{1-\mathrm{x}}$. Posteriormente, los átomos de nitrógeno se recombinan dentro de la capa compuesta, formando nitrógeno molecular, el cual es el responsable de los poros que aparecen en los lími-

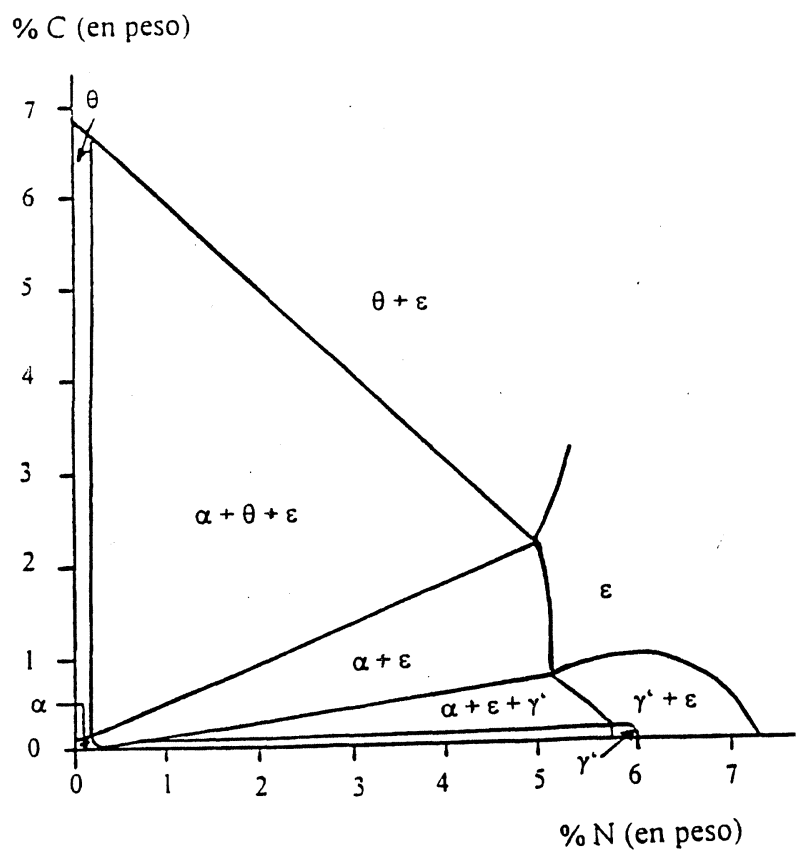

Fig. 1.- Diagrama de fases Fe-N-C a $570-580{ }^{\circ} \mathrm{C}$.

FIG. 1.- $\mathrm{Fe}-\mathrm{N}-\mathrm{C}$ phase diagram at $570-580^{\circ} \mathrm{C}$. tes de grano de la fase $\varepsilon$, su coalescencia forma canales hacia la superficie, a través de los cuales difundirá el carbono, con la consiguiente modificación de las condiciones de nitrocarburación. Otros autores han realizado numerosos estudios sobre la composición y estructura de la capa compuesta (4 y 5), y también de forma particular, tanto de la capa $\varepsilon$ (6) como de la capa $\gamma^{\prime}$ (7 y 8).

En una nitrocarburación prolongada crecen áreas enriquecidas en carbono y tiene lugar una nucleación de cementita $\left(\theta-\mathrm{Fe}_{3} \mathrm{C}\right)$. Estas formaciones de cementita coalescen y forman una capa continua. La capa nitrocarburada presentaría, por tanto, un aspecto tipo sandwich en la que aparecerían, desde la superficie hacia el material base, una capa de fase $\varepsilon$, relativamente porosa; luego, una de cementita; posteriormente, otra de fase $\varepsilon$ desprovista de poros, a continuación la fase $\gamma^{\prime} \mathrm{y}$, finalmente, el material base. El contenido de carbono en la superficie de la capa formada es relativamente bajo, creciendo hacia el interior de ésta y luego disminuyendo hacia el material base. El nitrógeno tiene un comportamiento opuesto (9), es decir, existe en mayor porcentaje en la superficie que en el interior de la capa nitrocarburada cercana al material base.

Las características de la zona de difusión son independientes del medio nitrocarburante y no puede distinguirse de la matriz original. La profundidad de esta zona de difusión disminuye con el nivel de formación de nitruros en el material; sin embargo, su dureza aumenta con el incremento del contenido en aleantes, debido a la precipitación de sus nitruros (10) como titanio, vanadio, cromo, etc.

Los aceros no aleados muestran una estructura estratificada de la capa nitrocarburada, apareciendo la fase $\varepsilon$ en la superficie y la fase $\gamma^{\prime}$ próxima al material base. En los materiales aleados, las fases $\varepsilon$ y $\gamma^{\prime}$, no están estructuradas en capas. En ambos casos, el porcentaje de fase $\varepsilon$ en la capa aumenta con el porcentaje de $\mathrm{CO}_{2}$ y con el de elementos de aleación (11 y 12).

Con la formación de este tipo de capas se consigue incrementar la resistencia al desgaste, mejorar la resistencia a la corrosión y reducir el coeficiente de rozamiento. Asimismo, se aumenta la resistencia a la fatiga después del temple, mediante la retención de nitrógeno en la zona de difusión (2).

\section{PARTE EXPERIMENTAL}

\subsection{Material}

El material utilizado fue un acero con la denominación AISI-SAE 8615 y cuya composición, en estado de recepción, se recoge en la tabla I. El material suministrado estaba conformado en barras recocidas de $15 \mathrm{~mm}$ de diámetro con una dureza de $250 \mathrm{HK}$ (237 HV). 
TABLA I.- Composición del acero AISI-SAE 8615, \%

TABLE I.-AISI-SAE 8615 steel composition, \%

\begin{tabular}{|c|c|c|c|c|c|c|c|c|}
\hline $\mathrm{Fe}$ & $\mathrm{C}$ & $\mathrm{Cr}$ & $\mathrm{Ni}$ & $\mathrm{Mo}$ & $\mathrm{Cu}$ & $\mathrm{Si}$ & $\mathrm{Mn}$ & $\mathrm{Bal}$ \\
\hline 96,92 & 0,124 & 0,55 & 0,46 & 0,17 & 0,26 & 0,19 & 1,21 & 0,116 \\
\hline
\end{tabular}

\subsection{Procedimiento}

Se cortaron probetas de $15 \mathrm{~mm}$ de longitud y se prepararon superficialmente mediante desbaste con papeles abrasivos hasta grado 1.000 , obteniéndose una rugosidad de $0,1-0,2 \mu \mathrm{m}(\mathrm{Ra})$. Las probetas se sometieron a un tratamiento de nitrocarburación gaseosa a $570{ }^{\circ} \mathrm{C}$ durante $3 \mathrm{~h}$, en un horno de $200 \mathrm{~L}$ de capacidad. La atmósfera fue generada por goteo a partir de una mezcla de urea y metanol. Finalmente, se realizó un temple en aceite, con el fin de congelar la estructura lograda durante el tratamiento termoquímico.

Con el fin de comprobar la influencia de la mezcla urea-metanol durante el proceso de nitrocarburación, se realizaron dos tipos de ensayos en los que se varió, en una primera serie, el porcentaje de mezcla $(5,10$ y $15 \%$ de urea en metanol), manteniendo constante la velocidad de flujo $(6 \mathrm{~mL} / \mathrm{min}) \mathrm{y}$, en una segunda serie, la velocidad de flujo (4, 6,8 y 8,8 $\mathrm{mL} / \mathrm{min}$ ) para un porcentaje constante del $15 \%$ de urea en la mezcla.

Las probetas, una vez tratadas en las distintas condiciones, se prepararon convencionalmente para realizar los análisis de composición, microdureza, y para su estudio metalográfico.

Las medidas de microdureza se realizaron tanto en el material en estado de recepción como en los tratados termoquímicamente, y se estudió su variación frente a las diferentes condiciones de ensayo. Las medidas se realizaron sobre las superficies transversales, utilizando un penetrador Knoop con una carga de $50 \mathrm{~g}$ y un tiempo de $20 \mathrm{~s}$.

La variación de composición en la zona nitrocarburada fue determinada mediante espectrometría de emisión. Tras realizar un primer análisis en la superficie, el espesor de la probeta se disminuye mediante desbaste, hasta la eliminación total de la huella producida por el electrodo. Se realizaron sucesivas medidas de composición, con las correspondientes disminuciones de espesor obtenidas por desbaste, hasta que se alcanzó el porcentaje de carbono igual al del material base.

La caracterización microestructural de las capas nitrocarburadas se realizó mediante microscopía óptica, sobre cortes transversales de las probetas ensayadas, utilizando como reactivo de ataque Nital ( $10 \% \mathrm{HNO}_{3}$ en etanol).

\section{RESULTADOS Y DISCUSIÓN}

\subsection{Ensayos con velocidad de flujo constante}

\subsubsection{Microestructura}

Si se comparan las estructuras de las probetas mostradas en las figuras $2-4$, obtenidas con una velocidad de flujo constante de $6 \mathrm{~mL} / \mathrm{min}$ de mezclas de urea-metanol, con porcentajes de urea del 5 , 10 y $15 \%$ respectivamente, se aprecia que, cuando se utiliza el menor porcentaje de urea, no aparece la capa de nitrocarburación de fase $\varepsilon$. Sin embargo, cuando este porcentaje es mayor (Figs. 3 y 4), se aprecia que no sólo aparece la capa de fase $\varepsilon$, sino que tiene un mayor espesor y es más homogénea. En todos los casos, la estructura del material base resulta prácticamente similar. Cuando la mezcla es del $10 \%$ en urea aparece capa blanca, aunque no de forma homogénea. Sin embargo, para la mezcla del $15 \%$ en urea, la capa blanca obtenida es homogénea y alcanza para las condiciones de ensayo un espesor de $2 \mu \mathrm{m}$.

\subsubsection{Medidas de microdureza y porcentajes en carbono}

En la figura 5 se muestran las variaciones del porcentaje de carbono y microdurezas, en función de la profundidad, para las distintas atmósferas de tratamiento.

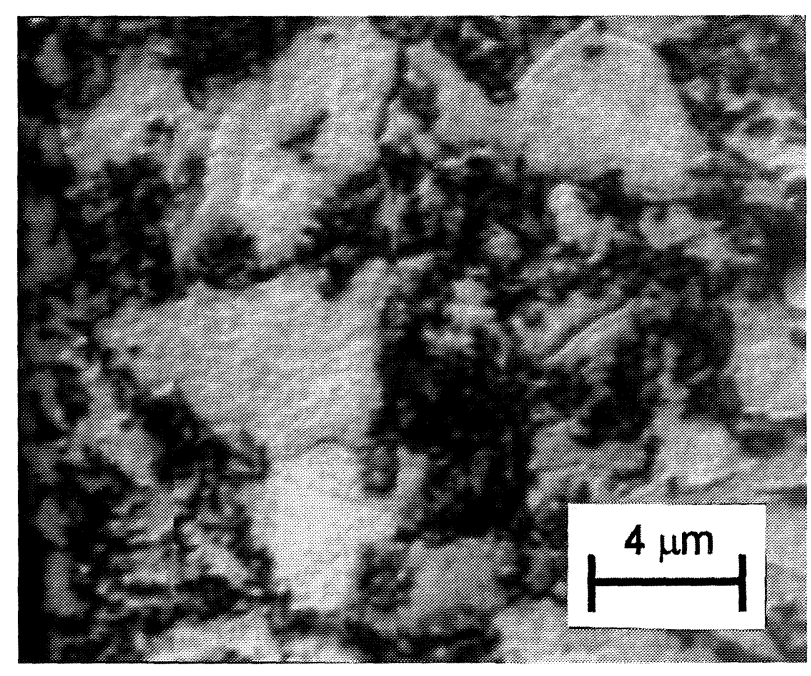

FIG. 2.- Microestructura del acero AISI-SAE 8615 nitrocarburado a $570{ }^{\circ} \mathrm{C}$ durante $3 \mathrm{~h}$ y templado en aceite. Atmósfera: mezcla $5 \%$ urea en metanol a $6 \mathrm{~mL} / \mathrm{min}$.

FIG. 2.- Microstructure of AISI-SAE 8615 steel nitrocarburized at $570{ }^{\circ} \mathrm{C}$ during $3 \mathrm{~h}$ and quenched in oil. Atmosphere: $5 \%$ urea/methanol at $6 \mathrm{~mL} / \mathrm{min}$. 


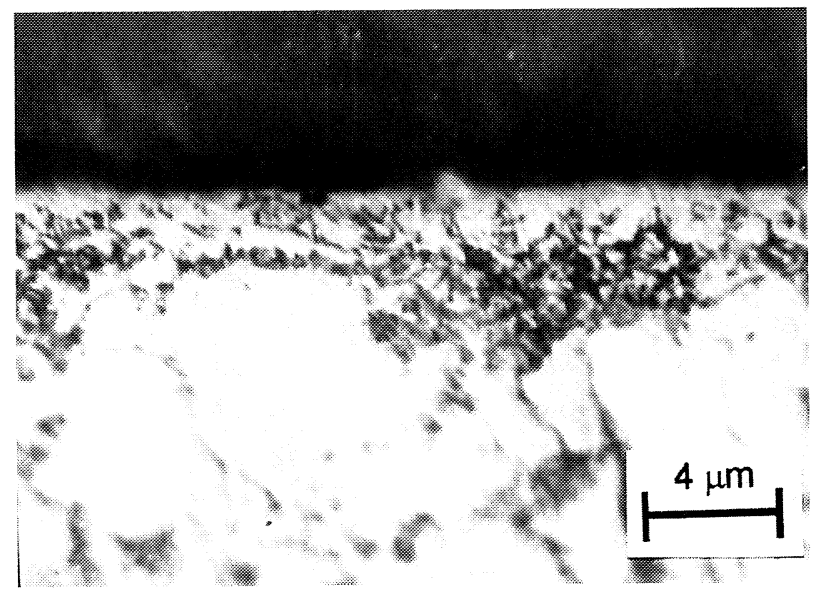

FIG. 3.- Microestructura del acero AISI-SAE 8615 nitrocarburado a $570{ }^{\circ} \mathrm{C}$ durante $3 \mathrm{~h}$ y templado en aceite. Atmósfera: mezcla $10 \%$ de urea en metanol a $6 \mathrm{~mL} / \mathrm{min}$.

FIG. 3.- Microstructure of AISI-SAE 8615 steel nitrocarburized at $570{ }^{\circ} \mathrm{C}$ during $3 \mathrm{~h}$ and quenched in oil. Atmosphere: $10 \%$ urea/methanol at $6 \mathrm{~mL} / \mathrm{min}$.

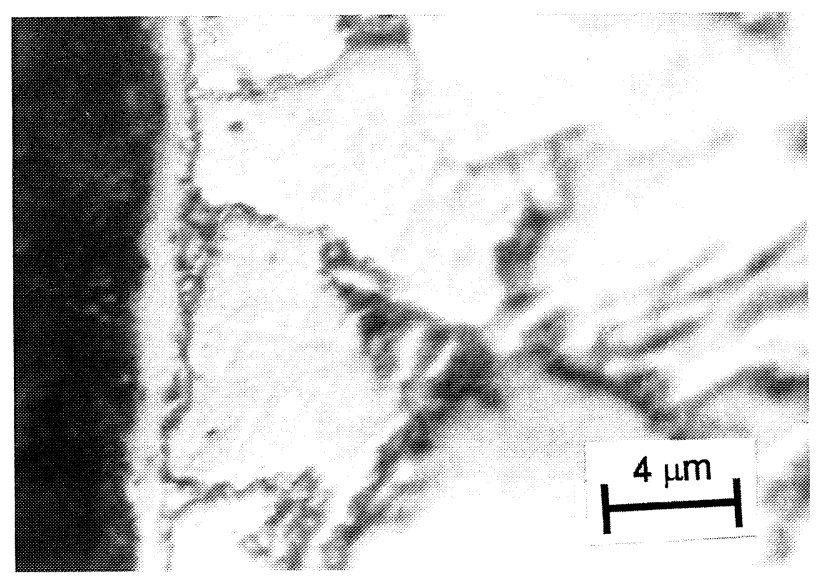

FIG. 4.- Microestructura del acero AISI-SAE 8615 nitrocarburado a $570{ }^{\circ} \mathrm{C}$ durante $3 \mathrm{~h}$ y templado en aceite. Atmósfera: mezcla $15 \%$ de urea en metanol a $6 \mathrm{~mL} / \mathrm{min}$. Espesor de capa blanca (fase $\epsilon$ ): $2 \mu \mathrm{m}$.

FIG. 4.- Microstructure of AISI-SAE 8615 steel nitrocarburized at $570{ }^{\circ} \mathrm{C}$ during $3 \mathrm{~h}$ and quenched in oil. Atmosphere: $15 \%$ urea/methanol at $6 \mathrm{~mL} / \mathrm{min}$. White layer thickness (phase $\epsilon$ ): $2 \mu \mathrm{m}$

Debido a las condiciones de ensayo, el espesor obtenido de la capa compuesta es muy pequeño y el contenido de carbono de la superficie no puede determinarse con gran precisión por el método analítico utilizado, ya que en las primeras disminuciones de sección entre medidas se pasa a analizar la zona de difusión. Por lo tanto, la sensibilidad para apreciar variaciones de composición dentro de la capa se ve disminuida. La variación del porcentaje de carbono a
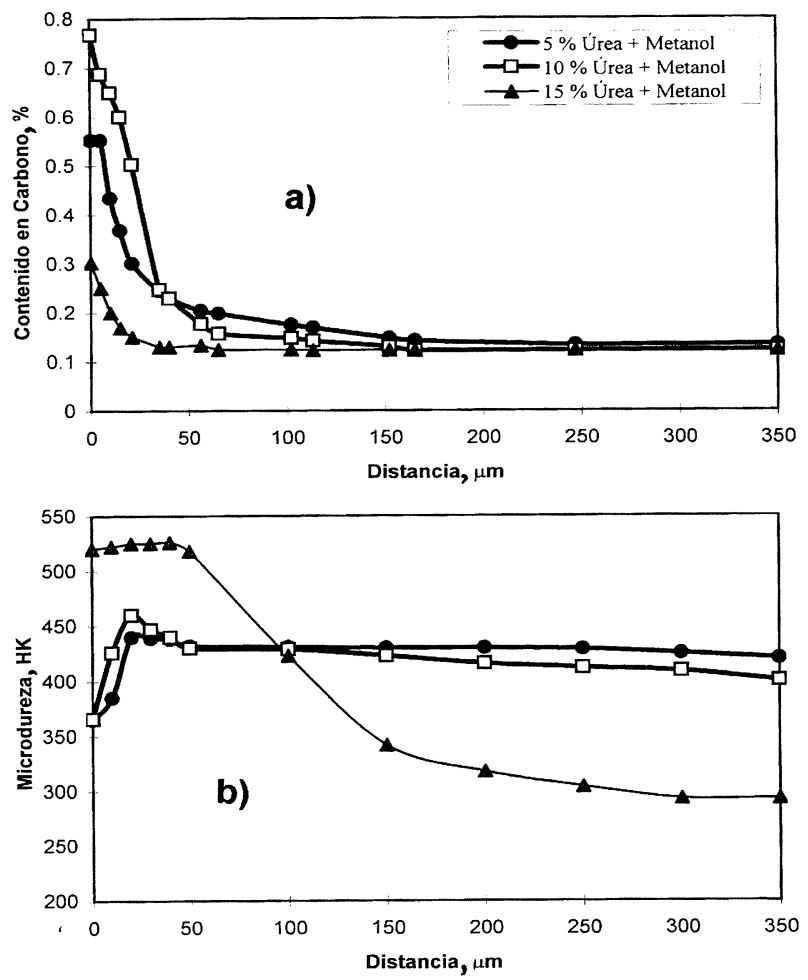

FIG. 5.- a) Contenido de carbono (\%), y b) valores de microdureza (HK) de la superficie nitrocarburada del acero AISI-SAE 8615, en función de los distintos porcentajes de urea en metanol de la atmósfera de nitrocarburación. Condiciones de tratamiento: Nitrocarburación a $570{ }^{\circ} \mathrm{C}$ durante $3 \mathrm{~h}$. Velocidad de flujo: $6 \mathrm{~mL} / \mathrm{min}$. Temple en aceite.

FIG. 5.- a) Carbon content (\%), and b) hardness values $(H K)$ and in the nitrocarburized surface of the AISI-SAE 8615 steel for the different urea percentages in methanol in the nitrocarburing atmosphere. Treatment conditions: Nitrocarburizing at $570{ }^{\circ} \mathrm{C}$ during $3 \mathrm{~h}$. Flow rate: $6 \mathrm{~mL} / \mathrm{min}$. Quenched in oil.

lo largo de la capa nitrocarburada y de la zona de difusión debería ser analizada utilizando otros métodos de análisis como EPMA, SIMMS o ESCA en cortes transversales. Sin embargo, se puede establecer el espesor de la zona de difusión en función de los valores de porcentaje de carbono obtenidos mediante espectroscopía de emisión y de los valores de microdureza en función de la distancia.

La zona de difusión de nitrógeno está situada entre la capa nitrocarburada y el material base. Esta zona de difusión es mayor cuanto menor es la capa compuesta, ya que el tener una capa compuesta grande y homogénea implica una barrera a la difusión de carbono y nitrógeno desde la superficie hacia el interior del material. En la figura 5a se observa la disminución del porcentaje de carbono a medida que se profundiza sobre el material. Cuando la capa nitrocarburada no se forma como se muestra 
en la figura 2 (5\% de urea en metanol), existe una mayor difusión de carbono en profundidad. El aumento en el porcentaje de urea implica un mayor contenido de carbono libre en la atmósfera del horno, que difunde y forma la capa deseada de fase $\varepsilon$, aunque no de forma homogénea en toda la superficie (10\% urea en metanol). En este caso, aunque el contenido de carbono es mayor en la cercanía de la superficie que en el ensayo anterior, al aumentar la profundidad se mantienen unos valores de concentración de carbono semejantes. Cuando se forma una capa homogénea de fase $\varepsilon$ con un espesor de 2 $\mu \mathrm{m}$ (15\% de urea en metanol) (Fig. 4), la difusión del carbono se ve dificultada como se muestra en la figura 5 a.

En la figura 5 b se representan los valores de microdureza toda la capa nitrocarburada. Al aumentar el contenido de urea en la mezcla, la dureza aumenta llegando a alcanzarse $526 \mathrm{HK}$ (498 HV) para un $15 \%$ de urea y una velocidad de flujo de 6 $\mathrm{mL} / \mathrm{min}$. Este aumento se debe al incremento del porcentaje de nitrógeno, formador de nitruros, retenido en la superficie.

Si se comparan los valores de microdureza para los distintos porcentajes de urea utilizados, se comprueba que existe un máximo de dureza, creciendo éste al aumentar el porcentaje de urea utilizada, lo cual se traduce en un aumento en la cantidad de nitruros formados. Sin embargo, la formación de una capa continua de fase $\varepsilon$ impide la difusión del nitrógeno y disminuye el espesor de la zona de difusión a medida que aumentamos el porcentaje de urea utilizado, lo que se refleja en una caída en el valor de microdureza a una menor distancia de la superficie. Cuando esta capa continua no se forma aparece descarburación en la superficie, lo que implica una menor dureza de la misma.

\subsection{Ensayos con velocidad de flujo variable}

\subsubsection{Microestructura}

Se ha utilizado un flujo de mezcla variable y un porcentaje constante de urea del $15 \%$, el cual fue elegido, debido a que las capas de nitrocarburación formadas son mayores y más homogéneas. Las figuras 6-8 muestran las microestructuras de las probetas sometidas al tratamiento termoquímico para velocidades de flujo de $4,6,8$ y $8,8 \mathrm{~mL} / \mathrm{min}$, respectivamente.

Cuando se utiliza una velocidad de flujo de 4 $\mathrm{mL} / \mathrm{min}$, se forman dos capas diferenciadas de la estructura del material base: la capa más externa (blanca), con un espesor del orden de 1,3 $\mu \mathrm{m}$, que se corresponde con la fase $\varepsilon$; una segunda capa (oscura), con un espesor aproximado de $4 \mu \mathrm{m}$, que

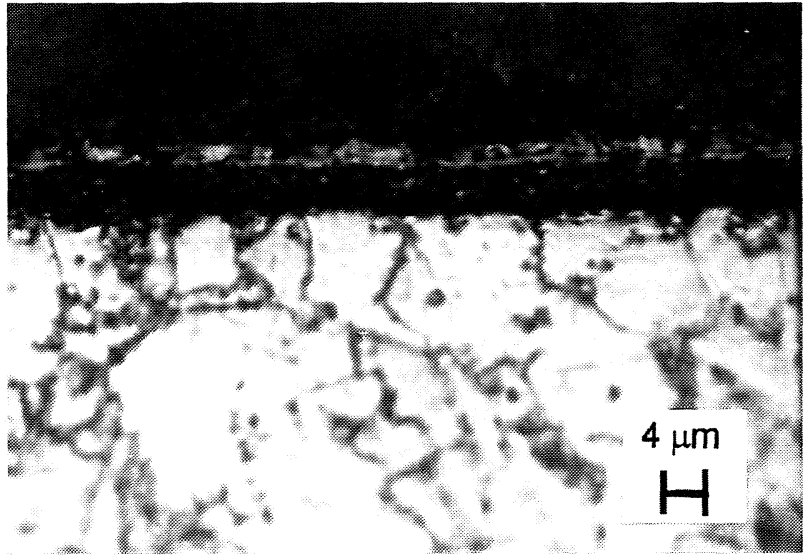

FIG. 6.- Microestructura del acero AISI-SAE 8615 nitrocarburado a $570{ }^{\circ} \mathrm{C}$ durante $3 \mathrm{~h}$ y templado en aceite. Atmósfera: mezcla $15 \%$ urea en metanol a $4 \mathrm{~mL} / \mathrm{min}$. Espesor de capa blanca (fase $\varepsilon$ ): 1,3 $\mu \mathrm{m}$.

Espesor de la capa oscura (fase $\gamma^{\prime}$ ): $4 \mu \mathrm{m}$.

FIG. 6.- Microstructure of AISI-SAE 8615 steel nitrocarburized at $570{ }^{\circ} \mathrm{C}$ during $3 \mathrm{~h}$ and quenched in oil. Atmosphere: $15 \%$ urea/methanol at $4 \mathrm{~mL} / \mathrm{min}$. White layer thickness (phase $\varepsilon$ ): 1,3 $\mu \mathrm{m}$.

Dark layer thickness (phase $\left.\gamma^{\prime}\right): 4 \mu \mathrm{m}$.

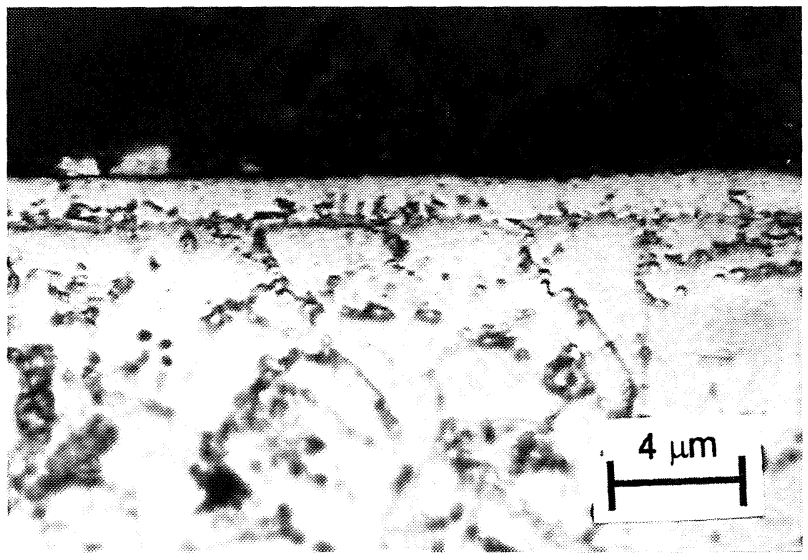

FIG. 7.- Microestructura del acero AISI-SAE 8615 nitrocarburado a $570{ }^{\circ} \mathrm{C}$ durante $3 \mathrm{~h}$ y templado en aceite. Atmósfera: mezcla $15 \%$ urea en metanol a $6,8 \mathrm{~mL} / \mathrm{min}$. Espesor de capa blanca (fase $\varepsilon$ ): $1,7 \mu \mathrm{m}$.

FIG. 7.- Microstructure of AISI-SAE 8615 steel nitrocarburized at $570{ }^{\circ} \mathrm{C}$ during $3 \mathrm{~h}$ and quenched in oil. Atmosphere: $15 \%$ urea/methanol at 6,8 $\mathrm{mL} / \mathrm{min}$. White layer thickness (phase $\varepsilon$ ): 1,7 $\mu \mathrm{m}$.

se corresponde con la fase $\gamma^{\prime} y$, finalmente, aparece la estructura del material base (Fig. 6). Cuando en el ensayo se utiliza una velocidad de flujo de 6,8 $\mathrm{mL} / \mathrm{min}$, el espesor formado de fase $\varepsilon$ aumenta hasta 1,7 $\mu \mathrm{m}$ (Fig. 7); sin embargo, la capa de fase $\gamma^{\prime}$ formada es insignificante $(<0,25 \mu \mathrm{m})$. Para una 


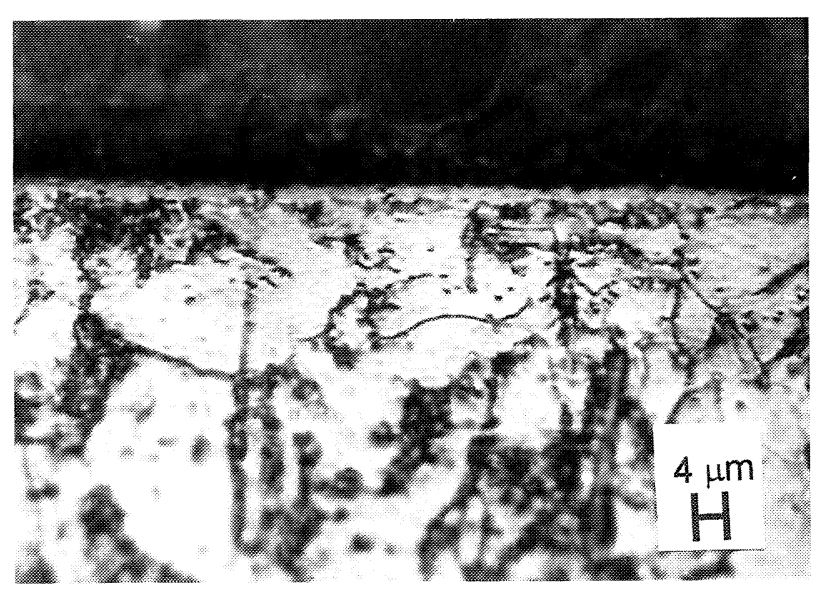

FIG. 8.- Microestructura del acero AISI-SAE 8615 nitrocarburado a $570{ }^{\circ} \mathrm{C}$ durante $3 \mathrm{~h}$ y templado en aceite. Atmósfera: mezcla $15 \%$ urea en metanol a $8,8 \mathrm{~mL} / \mathrm{min}$. Espesor de capa blanca (fase $\varepsilon$ ): $2 \mu \mathrm{m}$.

FIG. 8.- Microstructure of AISI-SAE 8615 steel nitrocarburazed at $570{ }^{\circ} \mathrm{C}$ during $3 \mathrm{~h}$ and quenched in oil. Atmosphere: $15 \%$ urea/methanol at 8,8 mL/min. White layer thickness (phase $\varepsilon$ ): $2 \mu \mathrm{m}$.

velocidad de flujo de $8,8 \mathrm{~L} / \mathrm{min}$, el espesor obtenido de fase $\epsilon$ es de $2 \mu \mathrm{m}$ y la fase $\gamma^{\prime}$ llega incluso a desaparecer por completo (Fig. 8), lo que demuestra que al aumentar la cantidad de mezcla aportada por unidad de tiempo, aumenta la cantidad de nitrógeno y carbono disponible en la atmósfera nitrocarburante y se favorece la formación de la capa $\varepsilon$ en detrimento de la capa $\gamma^{\prime}$, pues el rápido crecimiento de una capa continua y no porosa de fase $\varepsilon$ impide la difusión y, por tanto, el crecimiento de $\gamma^{\prime}$. En las tres condiciones de ensayo, las capas formadas son homogéneas en toda la superficie de la probeta.

\subsubsection{Medidas de microdureza y porcentajes en carbono}

De igual forma que en los casos anteriores, se muestran las variaciones de microdureza y de porcentaje de carbono de la superficie nitrocarburada (Fig. 9). La figura 9a muestra cómo en la capa de difusión, la cantidad de carbono difundido aumenta al aumentar la velocidad de flujo.

En la figura $9 \mathrm{~b}$ se representa la variación de los valores de microdureza frente a la profundidad. Hay que destacar que para una velocidad de flujo de 4 $\mathrm{mL} / \mathrm{mm}$ no se han podido obtener valores de microdureza fiables para la fase $\gamma^{\prime}$ debido a su gran fragilidad, por lo que no aparece el pico de máxima dureza.

De estas curvas, se deduce el efecto que tiene el espesor y la homogeneidad de la fase $\varepsilon$ formada en la evolución del proceso de nitrocarburación. Para
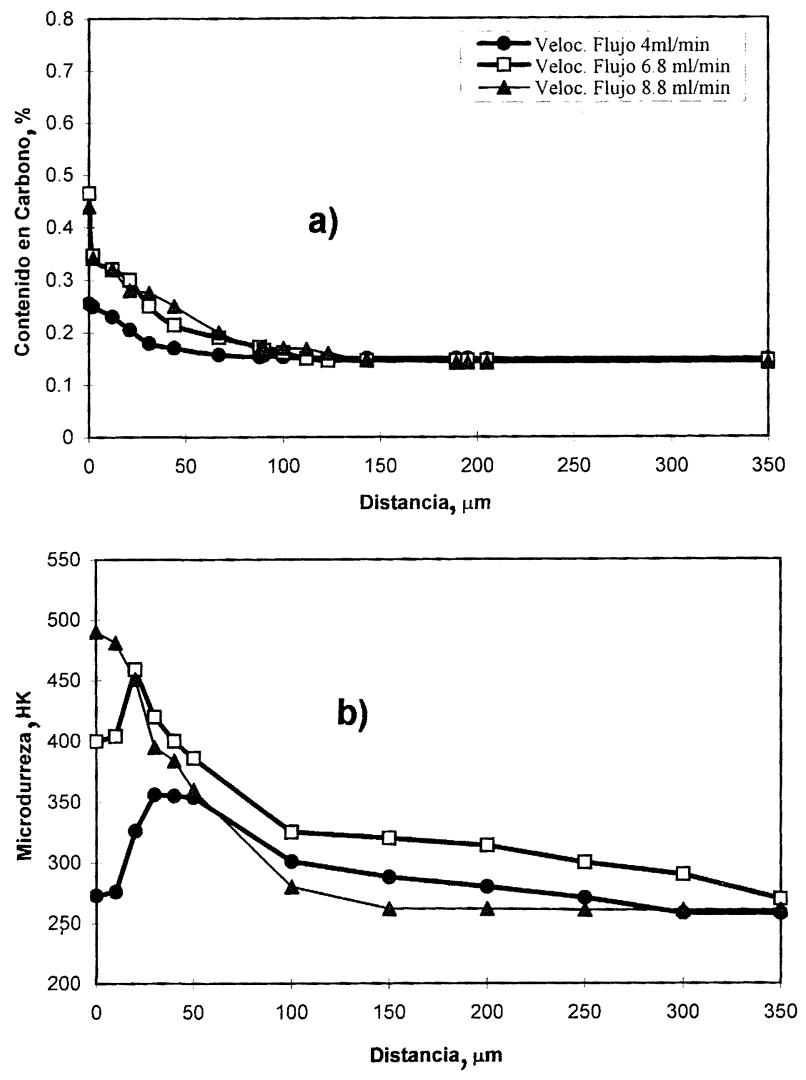

FIG. 9.- - a) Contenido de carbono (\%), y b) valores de microdureza (HK) de la superficie nitrocarburada de un acero AISI-SAE 8615, en función de las distintas velocidades de flujo de la mezcla de nitrocarburación. Condiciones de tratamiento: Nitrocarburación a $570{ }^{\circ} \mathrm{C}$ durante $3 \mathrm{~h}$, con un porcentaje del $15 \%$ de urea en metanol. Temple en aceite.

FIG. 9.- - a) Carbon content (\%), and b) hardness values $(H K)$ in the nitrocarburazed surface of the AISI-SAE 8615 steel for the different flow rates of nitrocarburing. Treatment conditions: Nitrocarburizing at $570{ }^{\circ} \mathrm{C}$ during $3 \mathrm{~h}$ with $15 \%$ urea in methanol. Quenched in oil.

velocidades de flujo bajas, la capa formada es más estrecha y porosa, la difusión no se ve impedida, la profundidad de la capa de difusión y la cantidad de nitruros formados aumenta con la cantidad de carbono y nitrógeno en la atmósfera del horno. Por otra parte, se aprecia una caída en los valores de microdureza en la superficie, debido a un proceso de descarburación. Existe una velocidad de flujo critica $(8,8 \mathrm{~mL} / \mathrm{min})$, a partir de la cual, la capa de fase $\varepsilon$ se hace muy gruesa y homogénea, lo que impide la difusión del nitrógeno. Aunque el valor de microdureza obtenido en la superficie es más alto que en los casos anteriores, éste decae rápidamente hasta valores comparables con los del material base. Por otro lado, no se produce descarburación del acero, pues se impide la reacción del 
carbono con el $\mathrm{CO}_{2}$ y $\mathrm{O}_{2}$ de la atmósfera del horno, como ocurre cuando se emplean velocidades de flujo menores de $8,8 \mathrm{~mL} / \mathrm{min}$.

\section{CONCLUSIONES}

- Es posible obtener capas nitrocarburadas de gran homogeneidad en un acero AISI-SAE 8615 utilizando porcentajes variables de urea en metanol.

- En función del flujo y de la cantidad de urea en la mezcla nitrocarburante, las capas obtenidas presentan distintos espesores y están constituidas por las fases $\varepsilon$ y $\gamma^{\prime}$. Cuando el porcentaje de urea es del $15 \%$ y el flujo de $8,8 \mathrm{~mL} / \mathrm{min}$ la fase $\gamma^{\prime}$ llega a desaparecer.

- El valor máximo de microdureza que se obtiene en la capa nitrocarburada es de 526 HK (498 $\mathrm{HV})$, cuando el flujo utilizado es de $6 \mathrm{~mL} / \mathrm{min}$ para un $15 \%$ de urea en metanol.

\section{REFERENCIAS}

(1) SPRoge, L. y Slycke, J.J. Heat Treat, 9, 1992: 105-112.

(2) Bell, T., Ferritic Gaseous Nitrocarburizing, American Society for Metales (1986), Metal Handbook, 9th Ed., vol 4, 264-269.

(3) Somers, M.A.J.y Mittemeijer, E.J., Mater. Sci. Forum, 10, 1992: 102-103

(4) Somers, M.A.J. y MitTemeiJer, E.J. Surf. Eng., 3, 1987 : 123-137.

(5) Slycke, J. y Sproge, L., Surf. Eng., 5, 1989: 125-140.

(6) El Ajaj, M., Foos, M., Michel, H. y Gantois, M., Scr. Metall., (17), 1983: 879-884.

(7) Somers, M.A.J. y Mittemeijer, E.J., Metall. Trans. A, (21A), 1990: 901-912.

(8) Somers, M.A.J. y Mittemeijer, E.J., Metall. Trans. A, (21A), 1990: 189-204.

(9) Krauss, G., Steels-Heat Treatment and Processing Principles, ASM 1989: 313.

(10) Honeycombe, R.W.K. y Bhadesshia, H.K.D.H., Steels. Microstructure and Properties, $2^{\mathrm{a}}$ ed., Ed. Edward Arnold, 1995: 60-82.

(11) Mittemeijer, E.J., Vogels, A.B.P. y Van der SchaAf, P.J.J. Mater. Sci., 15, 1980: 3.129-3.140.

(12) Rosso, M. y Scavino, G., Heat Treatment'87. The Institute of Metals, Londres, mayo 1987: 151-164. 